\begin{tabular}{|c|l|}
\hline Title & Hypernuclei and nuclear matter in a chiral SU (3) RMF model \\
\hline Author(s) & Tsubakihara, Kohsuke; Maekawa, Hideki; Ohnishi, A kira \\
\hline Citation & $\begin{array}{l}\text { The European Physical Journal A : Hadrons and Nuclei, 33/3), 295-298 } \\
\text { https://doi.org/L0.1140/epja/2007-10474-6 }\end{array}$ \\
\hline Issue Date & 2007-09 \\
\hline Doc URL & http://hdl.handle.net/2115/30179 \\
\hline Rights & The original publication is available at www.eurphys. org \\
\hline Type & article (author version) \\
\hline File Information & EPJA 33 3.pdf \\
\hline
\end{tabular}

Instructions for use 


\title{
Hypernuclei and nuclear matter in a chiral SU(3) RMF model
}

\author{
Kohsuke Tsubakihara, Hideki Maekawa and Akira Ohnishi \\ Department of Physics, Faculty of Science, Hokkaido University, Sapporo 060-0810, Japan. \\ e-mail: tsubaki@nucl.sci.hokudai.ac.jp
}

Received: date / Revised version: date

\begin{abstract}
We develop a chiral SU(3) RMF model for octet baryons, as an extension of the recently developed chiral SU(2) RMF model with logarithmic sigma potential. For $\Sigma$-meson coupling, strong repul$\operatorname{sion}(\mathrm{SR})$ and weak repulsion(WR) cases are examined in existing atomic shift data of $\Sigma^{-}$. In both of these cases, we need an attractive pocket of a few $\mathrm{MeV}$ depth around nuclear surface.
\end{abstract}

PACS. 21.65.+f Nuclear matter - 21.80.+a Hypernuclei

\section{Introduction}

In constructing the dense matter equation of state (EOS), it is strongly desired to respect both of hypernuclear physics information and chiral symmetry. Strangeness is expected to play a decisive role and the partial restoration of chiral symmetry would modify the hadron properties in dense matter. One of the promising approaches is to apply chiral symmetric relativistic mean field (RMF) models [1-7].

We have recently developed a chiral $\mathrm{SU}(2)$ symmetric RMF model [6] with logarithmic sigma potential in the form of $-\log \sigma$, which is derived in the strong coupling limit (SCL) of the lattice QCD [8]. In this model, the energy density in vacuum at zero temperature is evaluated in the mean field approximation as,

$$
\begin{aligned}
& U_{\sigma}=-a \log \left(\operatorname{det} M M^{\dagger}\right)+b \operatorname{tr}\left(M M^{\dagger}\right)+c_{\sigma} \sigma \\
& \quad \sim-2 a f_{\mathrm{SCL}}\left(\frac{\sigma}{f_{\pi}}\right)+\frac{1}{2} m_{\sigma}^{2} \sigma^{2}, \\
& f_{\mathrm{SCL}}(x)=\log (1-x)+x+\frac{x^{2}}{2}, \quad a=\frac{f_{\pi}^{2}}{4}\left(m_{\sigma}^{2}-m_{\pi}^{2}\right),
\end{aligned}
$$

where $M$ denotes the $\mathrm{SU}(2)$ meson matrix, $M=(\sigma+i \boldsymbol{\pi}$. $\tau) / \sqrt{2}$. In the second line of Eq. (1), $\sigma$ field is replaced with its fluctuation around the vacuum expectation value, $\sigma \rightarrow f_{\pi}-\sigma$. In this SCL model [6], we can describe bulk properties of finite nuclei, we have neither the chiral collapse at low densities [1] nor instability at large $\sigma$ [2], and the nuclear matter EOS is not very stiff [3]. Compared to previously proposed chiral RMF models $[4,5]$ and a more recently proposed one [7], this model has an advantage that the vacuum energy density is derived based on QCD.

It is straightforward to extend this chiral SU(2) RMF model to an $\mathrm{SU}(3)$ version which contains strangeness degrees of freedom. We expect that this extension enables us to get detailed information on $\Lambda, \Sigma$ and $\Xi$ hypernuclei.
In this paper, we determine the hyperon-meson coupling constants in this chiral $\mathrm{SU}_{f}(3) \mathrm{RMF}$ model by fitting existing data. We show that we can reproduce the separation energies of single $\Lambda$ hypernuclei $\left(S_{\Lambda}\right)$ [9] and the $\Lambda \Lambda$ bond energy $\left(\Delta B_{\Lambda \Lambda}\right)$ in ${ }_{\Lambda \Lambda}^{6} \mathrm{He}$ [10] by choosing the coupling constants appropriately in a reasonable parameter range. The EOS of symmetric matter is found to be softened by the scalar meson with hidden strangeness, $\zeta=\bar{s} s$, which couples with $\sigma$ through the determinant interaction. We also discuss the strength of repulsion in nuclear medium and attraction around nuclear surface in $\Sigma^{-}$-nucleus potential by comparing the calculated results with $\Sigma^{-}$atomic shift data [11].

\section{Chiral SU(3) RMF model}

In extending the chiral $\mathrm{SU}(2) \mathrm{RMF}$ model to $\mathrm{SU}(3)$, it is necessary to include mesons with hidden strangeness $(\bar{s} s)$ such as $\zeta$ and $\phi$ in addition to $\sigma, \omega$ and $\rho$. The chiral SU(2) RMF model [6] tells us the form of chiral potential of $\sigma$ and $\zeta$ by a simple extension written as,

$$
\begin{aligned}
U_{\sigma \zeta}= & -a \log \left(\operatorname{det} M M^{\dagger}\right)+b \operatorname{tr}\left(M M^{\dagger}\right) \\
& +c_{\sigma} \sigma+c_{\zeta} \zeta+d\left(\operatorname{det} M+\operatorname{det} M^{\dagger}\right),
\end{aligned}
$$

where the last term in rhs is introduced to take care of the $\mathrm{U}_{A}(1)$ anomaly. When the chiral symmetry is spontaneously broken and meson mass terms are generated, this effective interaction is written as,

$$
\begin{aligned}
U_{\sigma \zeta}= & -a\left[2 f_{\mathrm{SCL}}\left(\frac{\sigma}{f_{\pi}}\right)+f_{\mathrm{SCL}}\left(\frac{\zeta}{f_{\zeta}^{\prime}}\right)\right] \\
& +\frac{1}{2} m_{\sigma}^{2} \sigma^{2}+\frac{1}{2} m_{\zeta}^{2} \sigma^{2}+\xi_{\sigma \zeta} \sigma \zeta,
\end{aligned}
$$


where $f_{\zeta}^{\prime}=f_{\zeta}+m_{s}$ and $m_{s}$ is related to the strange quark mass. We have six parameters in this interaction $\left(a, b, c_{\sigma}\right.$, $c_{\zeta}, d$ and $\left.m_{s}\right)$, and five out of six are fixed by fitting experimental masses of $\pi, K$ and $\zeta$, and vacuum expectation values of $\sigma$ and $\zeta$. There remains only one parameter, $m_{\sigma}$. With this scalar meson effective interaction, the RMF Lagrangian is given as,

$$
\begin{aligned}
\mathcal{L} & =\mathcal{L}_{\text {Free }}\left(\psi_{i}, \bar{\psi}_{i}, \sigma, \zeta, \omega, \rho, \phi\right)+\mathcal{L}_{\mathrm{EM}}-U_{\sigma \zeta}+\frac{c_{\omega}}{4} \omega^{4} \\
& +\sum_{i} \bar{\psi}_{i}\left[g_{\sigma i} \sigma+g_{\zeta i} \zeta-\gamma_{\mu}\left(g_{\omega i} \omega^{\mu}+g_{\rho i} \rho^{\mu}+g_{\phi i} \phi^{\mu}\right)\right] \psi_{i},
\end{aligned}
$$

where the $\omega^{4}$ term is phenomenologically introduced to simulate the high density behavior of the vector self-energy in the RBHF theory as in Ref. [12].

In determining hyperon-vector meson couplings, we start from the $\mathrm{SU}_{f}(3)$ symmetric interaction,

$$
\begin{aligned}
\mathcal{L}_{\mathrm{BM}}=\sqrt{2}\left\{g_{s} \operatorname{tr}(M) \operatorname{tr}(\bar{B} B)\right. & +g_{1} \operatorname{tr}(\bar{B} M B) \\
& \left.+g_{2} \operatorname{tr}(\bar{B} B M)\right\} .
\end{aligned}
$$

Following the Okubo-Zweig-Iizuka (OZI) rule [13], we assume that nucleons do not couple with $\bar{s} s$ mesons $(\zeta$ and $\phi)$. Then there are two independent parameters, $g_{\omega N}$ and $g_{\rho N}$, and hyperon-vector meson coupling constants are found to be represented by $g_{\omega N}$ and $g_{\rho N}$ as follows,

$$
\begin{aligned}
& g_{\omega \Lambda}=\frac{5}{6} g_{\omega N}-\frac{1}{2} g_{\rho N}, \quad g_{\phi \Lambda}=\frac{\sqrt{2}}{3}\left(g_{\omega N}+3 g_{\rho N}\right) \\
& g_{\omega \Sigma}=g_{\rho \Sigma}=\frac{g_{\phi \Xi}}{\sqrt{2}}=\frac{1}{2}\left(g_{\omega N}+g_{\rho N}\right) \\
& g_{\omega \Xi}=g_{\rho \Xi}=\frac{g_{\phi \Sigma}}{\sqrt{2}}=\frac{1}{2}\left(g_{\omega N}-g_{\rho N}\right) .
\end{aligned}
$$

In the later discussion, we try to keep the above relations as far as possible.

In the scalar and pseudo scalar sector, it is necessary to include negative parity baryons or we only have Dtype when the chiral $\mathrm{SU}(3)$ symmetry is required $[5,18]$. This problem is out of the scope of this proceedings, and hyperon-scalar meson coupling constants are regarded as parameters. When the $\Lambda$-scalar meson couplings are obtained and $\mathrm{SU}_{f}(3)$ symmetry works also for scalar couplings, we can evaluate the $\Xi$-scalar couplings as,

$$
g_{\sigma \Xi}=\frac{2}{3} g_{\sigma N}-\frac{\sqrt{2}}{2} g_{\zeta \Lambda}, \quad g_{\zeta \Xi}=\frac{1}{3} g_{\sigma N}+\frac{\sqrt{2}}{2} g_{\zeta \Lambda} .
$$

\section{Nuclear matter and hypernuclei}

\subsection{Normal nuclei and nuclear matter}

In the present chiral RMF model, bulk properties of normal nuclei are well described, and these results are reported elsewhere. The strangeness degrees of freedom are found to soften the nuclear matter EOS, and thus have
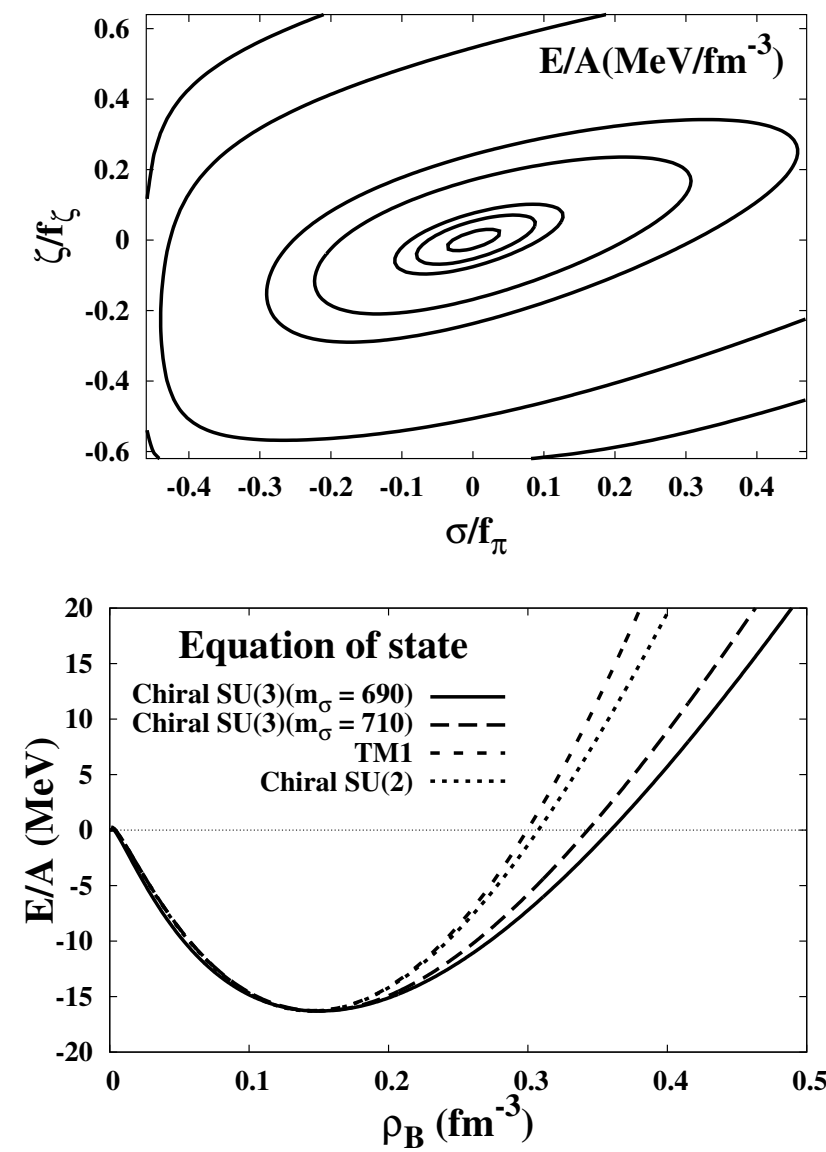

Fig. 1. Energy surface and EOS in chiral SU(3) model.

effects also on normal nuclei. The interaction in Eq. (3) contains the $\sigma \zeta$ mixing term, which gives rise to a correlation in $\sigma$ and $\zeta$ along the softest valley in the vacuum energy surface as shown in the upper panel of Fig. 1. Since the matter can evolve along this valley as the density increases, EOS is softened than in the chiral SU(2) RMF model [6], in which there is no $\zeta$ degree of freedom. The incompressibility is found to be $K \sim 220 \mathrm{MeV}$ when we fit the bulk properties of normal nuclei and nuclear matter saturation point, as shown in the lower panel of Fig. 1.

\section{$3.2 \Lambda$ hypernuclei}

Next we study $\Lambda$ hypernuclei with this chiral SU(3) RMF Lagrangian. There appear four additional parameters, $g_{\sigma \Lambda}$, $g_{\zeta \Lambda}, g_{\omega \Lambda}$ and $g_{\phi \Lambda}$. We fix the vector coupling constants, $g_{\omega \Lambda}$ and $g_{\phi \Lambda}$ by using the $\mathrm{SU}(3)$ symmetry relation in Eq. (6). Two remaining parameters are determined by fitting $S_{\Lambda}$ and $\Delta B_{\Lambda \Lambda}$ data. As shown in the upper panel of Fig. 2, we can explain $S_{\Lambda}$ nicely in a wide mass region by giving the $\Lambda$ potential depth around $30 \mathrm{MeV}$, which is represented by a linear combination of $g_{\sigma \Lambda}$ and $g_{\zeta \Lambda}$. By fitting $\Delta B_{\Lambda \Lambda}$ in ${ }_{\Lambda \Lambda}^{6}$ He simultaneously with $S_{\Lambda}$, both of $g_{\sigma \Lambda}$ and $g_{\zeta \Lambda}$ are determined as shown in Fig. 2 . 

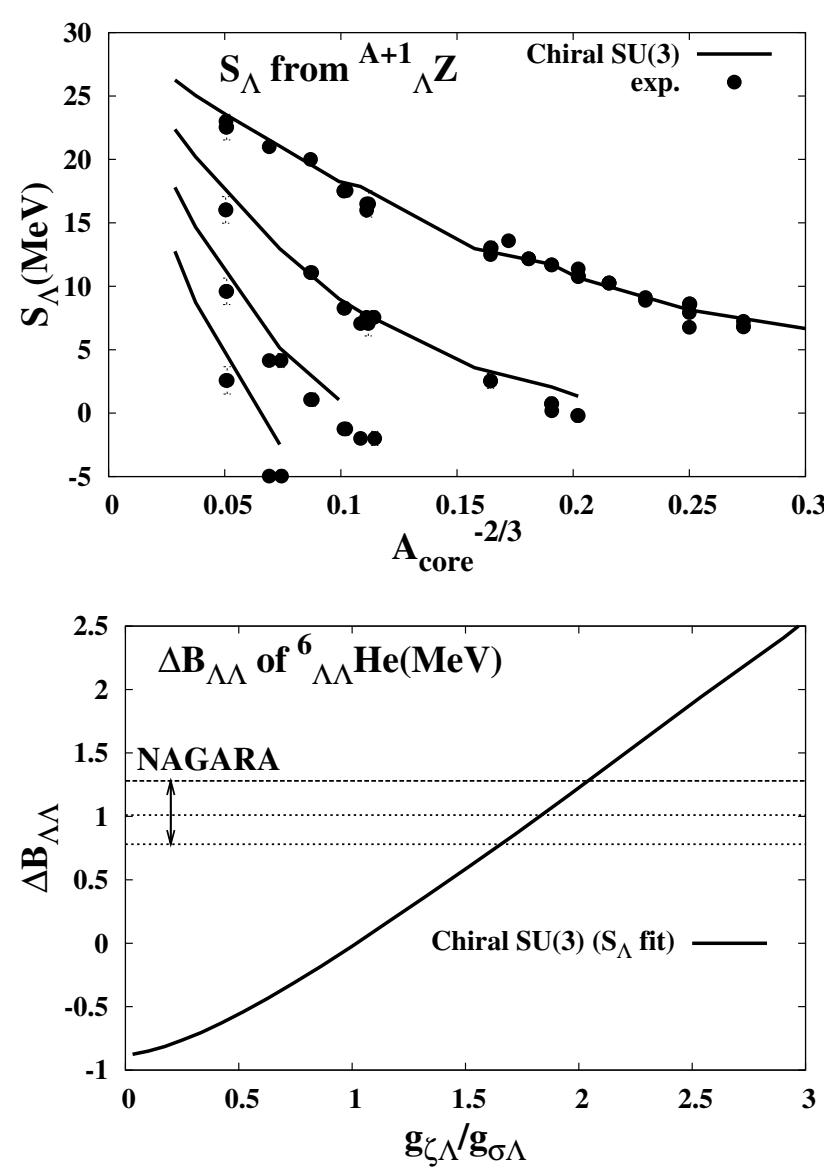

Fig. 2. $\Lambda$ separation energy and $\Delta B_{\Lambda \Lambda}$ of ${ }_{\Lambda \Lambda}^{6} \mathrm{He}$.

\section{$3.3 \Sigma$ hyper atom}

Recent analyses of quasi-free $\Sigma^{-}$production spectra [14, 15] suggest that $\Sigma^{-}$-nucleus potential should be repulsive in nuclear medium. On the other hand, $\Sigma^{-}$-nucleus potential needs to possess a few $\mathrm{MeV}$ attractive pocket around nuclear surface to explain $\Sigma^{-}$atomic shift data $[16,17]$. Here we would like to extract $\Sigma$-meson coupling constants which explain $\Sigma^{-}$atomic shifts. In the present RMF model, we have four additional parameters for $\Sigma, g_{\sigma \Sigma}, g_{\zeta \Sigma}, g_{\omega \Sigma}$ and $g_{\rho \Sigma}$. First we set $g_{\omega \Sigma}$, which determines the strength of repulsion in nuclear medium. We have examined two cases. (i) Strong Repulsion (SR) case: From the flavor $\mathrm{SU}(3)$ symmetry and OZI rule, $g_{\omega \Sigma}$ is given as $g_{\omega \Sigma}=$ $\left(g_{\omega N}+g_{\rho N}\right) / 2 \sim 2 g_{\omega N} / 3$. (ii) Weak Repulsion (WR) case: $g_{\omega \Sigma} \sim g_{\omega N} / 3$ which is also adopted in Ref. [17]. Secondly, scalar meson couplings $\left(g_{\sigma \Sigma}\right.$ and $\left.g_{\zeta \Sigma}\right)$, which determine the attractive pocket depth around nuclear surface, are chosen so as to reproduce atomic shifts of symmetric $N=Z$ core nuclei $(\mathrm{O}, \mathrm{Si}, \mathrm{S})$. Finally, $g_{\rho \Sigma}$ is adjusted to get a correct atomic shift in $\mathrm{Pb}$.

In Fig. 3, we show calculated atomic shifts and conversion widths of $\mathrm{O}, \mathrm{Mg}, \mathrm{Al}, \mathrm{Si}, \mathrm{S}, \mathrm{W}$ and $\mathrm{Pb}$ for $n=$ $4 \rightarrow 3(\mathrm{O}), n=5 \rightarrow 4(\mathrm{Mg}, \mathrm{Al}, \mathrm{Si}$ and $\mathrm{S})$ and $n=10 \rightarrow$ $9(\mathrm{~W}$ and $\mathrm{Pb})$ transitions. Atomic shift results are in good agreement except for $\mathrm{W}$ and the total $\chi^{2} /$ dof is around
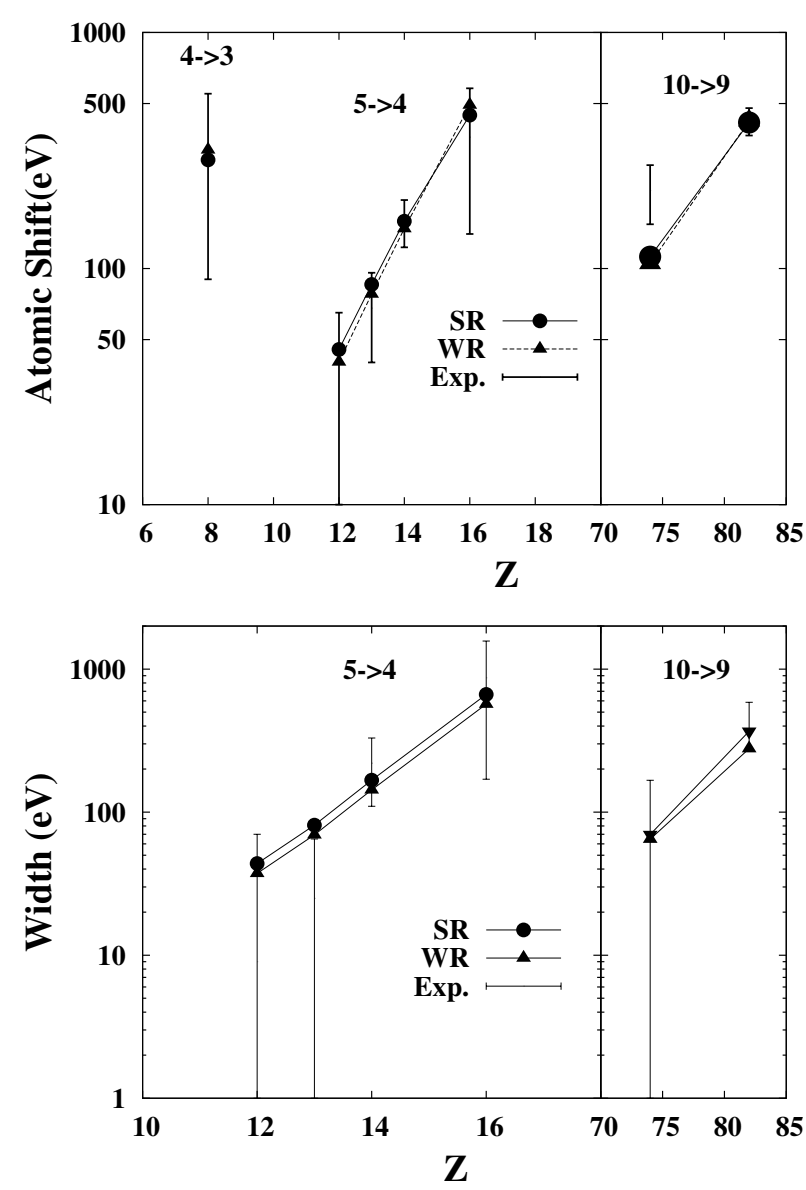

Fig. 3. Atomic shift and conversion width of $\Sigma^{-}$.

1.3. The conversion width is calculated as the expectation value of $\operatorname{Im} V_{\mathrm{opt}}=t \rho_{p}$. Imaginary parts are found to be $-15 \sim-20 \mathrm{MeV}$.

\section{Summary and conclusion}

We have developed a chiral $\mathrm{SU}(3)$ relativistic mean field (RMF) model with a logarithmic chiral potential for $\sigma$ and $\zeta(=\bar{s} s)$ mesons derived in the strong coupling limit of lattice QCD [8], as an extension of the chiral SU(2) RMF model [6]. The chiral symmetry and the mass generation by the spontaneous chiral symmetry breaking give severe constraints on parameters. After fitting several meson masses and vacuum expectation values, $m_{\sigma}$ is left unfixed in this chiral potential. Nucleon parameters $(N$ meson coupling constants, $m_{\sigma}$ and the coefficient of $\omega^{4}$ term) are determined to reproduce the vacuum nucleon mass, the nuclear matter saturation point, and bulk properties (binding energies and charge rms radii) of normal nuclei from $\mathrm{C}$ to $\mathrm{Pb}$ isotopes. $\Lambda$-meson coupling constants are determined by fitting hypernuclear data ( $\Lambda$ separation energies $S_{\Lambda}$ and $\Lambda \Lambda$ bond energy $\left.B_{\Lambda \Lambda}\right)$ under the constraints of $\mathrm{SU}_{f}(3)$ symmetry for vector couplings.

By fitting the $\Sigma^{-}$atomic shifts, we find that the attractive pocket in the $\Sigma$-nucleus potential around the nu- 

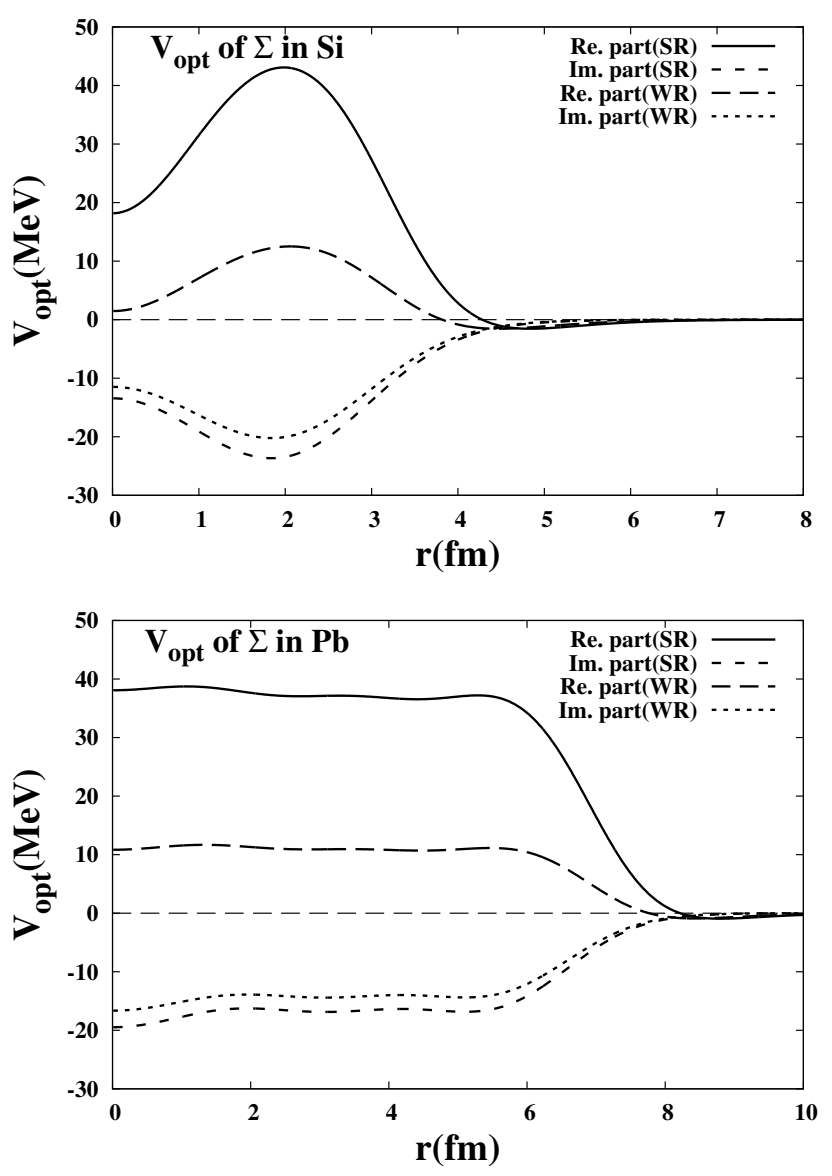

Fig. 4. Re. part and Im. parts of optical potentials of $\Sigma^{-}$in SR and WR cases.

clear surface should have a few MeV depth. The conversion widths of $\Sigma^{-}$atom are well described with the imaginary part of the optical potential in the form of $\operatorname{Im} V_{\mathrm{Opt}}=t \rho_{p}$, and the strengths are found to be $-15 \sim-20 \mathrm{MeV}$. These results are consistent with the previous RMF analysis [17].

We have tried to keep the $\mathrm{SU}_{f}(3)$ relations in the baryon-vector meson coupling constants as far as possible, and these relations seem to work well for $\Lambda$ hypernuclei. However, we have to break the $\mathrm{SU}_{f}(3)$ relation for $g_{\rho \Sigma}$ to reproduce atomic shift data at $N>Z$. It is suggested that the short-range repulsion is strong in $\Sigma N$ interaction due to the Pauli blocking between quarks. Therefore, the present result may indicate that we cannot describe $\Sigma^{-}$-nucleus potential properly in the chiral SU(3) RMF, which should be applicable to hadronic interactions, and that it is necessary to include the short-range repulsion from quarks. While we have this conceptual problem, we have now a chiral SU(3) RMF model, which can describe nuclear matter, finite normal nuclei, single and double $\Lambda$ hypernuclei and $\Sigma^{-}$atom.

It is desired to check the consistency between the present results and quasi-free spectrum analyses. We have investigated $\Sigma^{-}$quasi-free spectrum with DWIA+Local Optimized Fermi Average t-matrix [15]. With this method, it would be possible to judge whether $\Sigma^{-}$repulsion should be strong or relatively weak. It is also interesting to investigate $\Xi$ hypernuclei and hyperatoms. If the $\mathrm{SU}_{f}(3)$ relations in Eqs. (8) and (9) approximately hold in $\Xi$-meson couplings, we have smaller ambiguities in the $\Xi$-nucleus potential. Predictions along this line are in progress.

\section{Acknowledgment}

This work is supported in part by the Ministry of Education, Science, Sports and Culture, Grant-in-Aid for Scientific Research under the grant numbers, 15540243 and 1707005 .

\section{References}

1. J. Boguta, Phys. Lett. B 120, (1983) 34; Phys. Lett. B 128, (1983) 19.

2. T. Matsui and B. D. Serot, Ann. Phys. 144, (1982) 107; P. Sahu and A. Ohnishi, Prog. Theor. Phys. 104, (2000) 1163 [arXiv:nucl-th/0007068].

3. Y. Ogawa H. Toki, S. Tamenaga, H. Shen, A. Hosaka, S. Sugimoto and K. Ikeda, Prog. Theor. Phys. 111, (2004) 75 [arXiv:nucl-th/0312042].

4. R. J. Furnstahl and B. D. Serot, Phys. Lett. B 316, (1993) 12; E. K. Heide, S. Rudaz, and P. J. Ellis, Nucl. Phys. A 571, (1994) 713. [arXiv:nucl-th/9308002].

5. P. Papazoglou et al., Phys. Rev. C 57, (1998) 2576 [arXiv:nucl-th/9706024].

6. K. Tsubakihara and A. Ohnishi, arXiv:nucl-th/0607046.

7. D. Zschiesche, L. Tolos, J. Schaffner-Bielich and R. D. Pisarski, arXiv:nucl-th/0608044.

8. N. Kawamoto and J. Smit, Nucl. Phys. B 190, (1981) 100; N. Kawamoto, K. Miura, A. Ohnishi and T. Ohnuma, Phys. Rev. D 75, (2007) 014502 [arXiv:hep-lat/0512023].

9. R. E. Chiren, Nucl. Phys. A 479 (1988), 705; P. H. Pile et al., Phys. Rev. Lett. 17, (1991) 2535; T. Hasegawa et al., Phys. Rev. C 53, (1996) 1210;

10. H. Takahashi et al., Phys. Rev. Lett. 87, (2001) 212502.

11. C. J. Batty et al., Phys. Lett. B 74, (1978) 27;

R. J. Powers et al., Phys. Rev. C 47, (1993) 1263.

12. Y. Sugahara and H. Toki, Nucl. Phys. A 579, (1994) 557.

13. S. Okubo, Phys. Lett. 5, (1963) 1975; G. Zweig, Developments in the Quark Theory of Hadrons (Hadronic Press, Massachusetts, 1980); J. Iizuka, Prog. Theor. Phys. Suppl. 37, (1966) 38.

14. M. Kohno, Y. Fujiwara, Y. Watanabe, K. Ogata and M. Kawai, Prog. Theor. Phys. 112 (2004), 895 [arXiv:nuclth/0410073]; T. Harada and Y. Hirabayashi, Nucl. Phys. A 759, (2005) 143.

15. H. Maekawa, K. Tsubakihara and A. Ohnishi, in this proceedings [arXiv:nucl-th/0701066].

16. C. J. Batty, E. Friedman and A. Gal, Phys. Lett. B 335, (1994) 273.

17. J. Mares, E. Friedman, A. Gal and B. K. Jennings, Nucl. Phys. A 594, (1995) 311 [arXiv:nucl-th/9505003].

18. G. A. Christos, Phys. Rev. D 35, (1987) 330; A. Ohnishi and K. Naito, Proc. of VIII Int. Conf. on Hypernuclear \& Strange Particle Physics, Jefferson Lab., Newport News, Virginia, USA, Oct. 14-18, 2003 (unpublished). 